\title{
High Sensitivity C-Reactive Protein as an Independent Predictor of Progressive Myocardial Functional Deterioration: The Multi- Ethnic Study of Atherosclerosis
}

\author{
Eui-Young Choi, MD, PhD ${ }^{a, b}$, Raymond T. Yan, MDa, Veronica R.S. Fernandes, MD $^{c}$, Anders \\ Opdahl, MDa , Antoinette S. Gomes, MDd, Andre L.C. Almeida, MDa, Colin O. Wu, PhD ${ }^{\mathrm{e}}$, \\ Kiang Liu, PhD ${ }^{f}$, Jeffrey J. Carr, MDg, Robyn L. McClelland, PhD $^{h}$, David A. Bluemke, MD, \\ PhDi, and Joao A. C. Lima, MDa \\ aJohns Hopkins University \\ bYonsei University College of Medicine, Seoul, South Korea \\ cBeth Israel Deaconess Medical Center \\ dUCLA School of Medicine \\ eNational Heart, Lung and Blood Institute \\ ${ }^{f}$ Northwestern University Medical School \\ gWake Forest University \\ hUniversity of Washington \\ 'National Institutes of Health
}

\section{Abstract}

Background-Systemic inflammation has been linked to the development of heart failure in population studies including MESA (Multi-Ethnic Study of Atherosclerosis) but little evidence exists regarding potential mechanism of this relationship. In this study, we used longitudinal MRI follow-up analysis to examine whether C-reactive protein (CRP) levels relate to progressive myocardial functional deterioration as a potential mechanism of incident heart failure.

\begin{abstract}
Methods-Regional myocardial functional data from MESA participants who had baseline CRP measurement and also underwent tagged cardiac MRI both at baseline and at five-year follow-up were analyzed. Left ventricular (LV) midwall and mid-slice peak circumferential strain (Ecc), of which a more negative value denotes stronger regional myocardial function, was measured. Ecc change was calculated as the difference between baseline and follow-up Ecc.
\end{abstract}

Results-During the follow-up period, participants $(n=785)$ with elevated CRP experienced a decrease in strain, independent of age, gender and ethnicity $(B=0.081 ; \Delta E c c$ change per $1 \mathrm{mg} / \mathrm{L}$ CRP change, 95\% CI 0.036-0.126, $\mathrm{p}<0.001$, Model 1), and additionally beyond systolic blood

(C) 2012 Mosby, Inc. All rights reserved.

Corresponding author: Joao A.C. Lima, MD, FACC, Professor of Medicine, Johns Hopkins Hospital, 600 North Wolfe Street, Blalock 524D1, Baltimore, MD 21287, jlima@jhmi.edu.

Disclosures

The authors are solely responsible for the design and conduct of this study, all study analyses and drafting and editing of the paper.

Publisher's Disclaimer: This is a PDF file of an unedited manuscript that has been accepted for publication. As a service to our customers we are providing this early version of the manuscript. The manuscript will undergo copyediting, typesetting, and review of the resulting proof before it is published in its final citable form. Please note that during the production process errors may be discovered which could affect the content, and all legal disclaimers that apply to the journal pertain. 
pressure, heart rate, diabetes, smoking status, body mass index, current medication and glomerular filtration rate $(\mathrm{B}=0.099,0.052-0.145, \mathrm{p}<0.001$, Model 2$)$. The relationship remained statistically significant after further adjustment for LV mass, coronary calcium score and interim clinical coronary events ( $\mathrm{B}=0.098,0.049-0.147, \mathrm{p}<0.001$, Model 3).

Conclusion-Higher CRP levels are related to progressive myocardial functional deterioration independent of subclinical atherosclerosis and clinical coronary events in asymptomatic individuals without previous history of heart disease.

\section{Keywords}

inflammation; myocardial function; magnetic resonance imaging

\section{Introduction}

Systemic inflammation has been shown to be related to hospitalization and mortality in patients with heart failure (HF). ${ }^{1}$ High sensitivity C-reactive protein (CRP) is a biomarker of inflammation which has been associated with the development of symptomatic HF in largescale population studies. Indeed, several prior reports have demonstrated a clear link between elevated levels of CRP and the development of HF, even among asymptomatic individuals. ${ }^{2,3}$ Among potential mechanisms, myocardial infarction caused by coronary arterial plaque rupture due to the local activation of pro-inflammatory cytokines is a well known determinant of incident HF. ${ }^{4,5}$ However, the greater incidence of HF among patients with elevated CRP has been observed to be in large part independent of interim myocardial infarction in previous population studies, including the Multi-Ethnic Study on Atherosclerosis (MESA). ${ }^{3}$ Therefore, the mechanisms by which higher systemic inflammatory markers contribute to the development of HF have not been fully elucidated. Basic research suggests that direct myocardial functional depression may be induced by inflammatory cytokines. ${ }^{6}$ In this regard, recent population studies have shown that CRP levels are independently related to regional myocardial dysfunction, although causation could not be ascertained in such cross sectional analyses. ${ }^{5}$ Until now, there have been no large-scale longitudinal follow-up studies designed to evaluate the effects of systemic inflammation on progression of regional myocardial dysfunction in community-based populations.

Myocardial circumferential strain, defined as proportional myocardial deformation during the cardiac cycle, can be accurately measured regionally by tagged magnetic resonance imaging (MRI), and has been validated and used as a reference index of regional myocardial function. ${ }^{7}$ Therefore, in this MESA ancillary study, we used serial cardiac MRI tagging examinations to determine whether elevated CRP can predict progressive regional myocardial functional deterioration independent of interim cardiovascular events, in a multiethnic population without clinically apparent cardiovascular disease at baseline.

\section{Methods}

\section{Study population}

The MESA is a prospective study designed to evaluate mechanisms that underlie the development and progression of subclinical cardiovascular diseases among asymptomatic individuals of the general population. The details of the MESA study design have been previously described. ${ }^{8}$ In short, 6,814 American men and women, 45 to 85 years of age, free of known cardiovascular disease, of four self-reported ethnicities ("non-Hispanic white," "African American," "Hispanic," and "Chinese American") were enrolled by six participating centers in the United States. On entry, all participants underwent an extensive 
evaluation that consisted of clinical questionnaires, physical examination, and laboratory tests. Individuals with symptomatic cardiovascular disease were excluded. Computed tomography (CT) scanning of the chest was performed either with ECG-triggered electron beam CT, or multidetector CT system. Cardiac MRI was done in 5,098 participants and as an ancillary study, tagged MRI was performed at baseline and repeated at MESA 4 in part of them. The institutional review boards in each of the participating centers approved the study protocol and informed consent was obtained from each participant.

\section{High sensitive-CRP concentrations}

Serum level of CRP was measured at the baseline MESA examination as a marker of systemic inflammation. Level of CRP was measured using the BNII nephelometer (N HighSensitivity CRP; Dade Behring, Deerfield, IL, USA). Analytical intra-assay coefficients of variation (CVs) of CRP ranged from $2.3 \%$ to $4.4 \%$ and interassay $\mathrm{CVs}$ ranged from $2.1 \%$ to $5.7 \%$.

\section{Baseline and follow-up tagged MRI studies}

The detailed protocol for tagged MRI studies has been previously described. ${ }^{9}$ The same tagged MRI protocol was applied at baseline and at follow-up scanning. Images were acquired by whole-body scanners (1.5 CVi, General Electric Medical Systems, Waukesha, WI, USA and Sonata/Symphony Siemens Medical Solutions, Erlangen, Germany) using ECG-triggered segmented $k$-space fast spoiled gradient-echo pulse sequence during breath holds. After completing the standard protocol, three tagged short-axis slices (base to apex) were obtained. Parallel striped tags were prescribed in two orthogonal orientations $\left(0^{\circ}\right.$ and $90^{\circ}$ ) using spatial modulation of magnetization. Parameters for tagged images were: field of view $40 \mathrm{~cm}$; slice thickness 7 to $8 \mathrm{~mm}$; repetition time $6 \mathrm{~ms}$; echo time $3.0 \mathrm{~ms}$; flip angle $10^{\circ}$ to $12^{\circ}$; phase encoding views 128 with 6 phase encoding views per segment; temporal resolution $35 \mathrm{~ms}$; tag spacing $7 \mathrm{~mm}$. The LV mass, LV end-diastolic volume, and LV ejection fraction were determined for each participant using dedicated, commercially available software (MASS, version 4.2, Medis, Leiden, The Netherlands) as previously described. ${ }^{10}$

\section{Myocardial strain analysis}

Myocardial systolic circumferential strain (Ecc) was measured to evaluate regional myocardial function. Short-axis tagged slices were analyzed using the HARP method (Diagnosoft, Palo Alto, California) which enables fast determination of myocardial strain during the cardiac cycle. ${ }^{11}$ Ecc was determined in four wall segments (anterior, lateral, inferior, and septal) from the LV midwall layer ${ }^{9,12}$ using MATLAB ${ }^{\circledR}$ software (The MathWorks, MA, USA) (figure 1). By convention, systolic Ecc is normally negative denoting circumferential shortening and higher Ecc values reflect decreased regional function (for example, Ecc $=-12 \%$ reflects lower regional function when compared with $\mathrm{Ecc}=-18 \%$ ). Segments without well defined peak strain curves due to significant noise were excluded. Intraclass correlation coefficients for interobserver and intraobserver agreement for peak systolic midwall Ecc were 0.81 and 0.84 respectively. ${ }^{9}$ Peak global systolic strain was defined as the peak midwall Ecc averaged across all mid-ventricular segments. Peak Ecc change during the follow-up period was calculated by subtracting peak global mid-LV slice Ecc at baseline from Ecc at the follow-up exam to reduce mismatching between the two exams.

\section{Interim cardiovascular events}

At intervals between nine to 12 months, interviewers contacted with each participant by telephone to document interim hospital admissions, cardiovascular outpatient diagnoses or 
deaths. Interviewers took history of angina followed by revascularization, stroke, peripheral artery disease, myocardial infarction, resuscitated cardiac arrest, and death from coronary disease. If interviewers failed to contact participants directly, they contacted family members. For participants who had died of cardiovascular causes outside the hospital, interviews were conducted with the next of kin and copies of death certificates were requested. To verify self-reported diagnoses, study personnel requested copies of all medical records for all hospitalizations and outpatient cardiovascular diagnoses. Hospital records were obtained for an estimated $98 \%$ of cardiovascular events requiring hospitalization and some information was available for $95 \%$ of outpatient diagnostic encounters. The MESA coordinating center collated the abstracted or original end point records and sent them to two paired cardiologists and cardiovascular epidemiologists for independent end point classification and assignment of incidence dates. If disagreements persisted after review and adjudication, a full Mortality and Morbidity Review Committee made the final classification. Reviewers assigned a diagnosis of myocardial infarction based on a combination of symptoms, ECG findings, and cardiac biomarker levels. The definition of angina leading to hospitalization was adapted from the Women's Health Initiative criteria. Reports of percutaneous coronary intervention and bypass surgery were also obtained from medical records.

\section{Statistical analysis}

Pearson's correlation analysis was performed to evaluate relationships between continuous variables. Comparisons of continuous variables across CRP quartile groups were performed using ANOVA-F analysis or Kruskal-Wallis tests. Comparisons of discrete variables were performed by chi-square tests while comparisons of Ecc values between two exams were performed using paired t-tests. For categorical analyses of change in Ecc, we defined a "definite" Ecc deterioration group as participants who had $\Delta$ Ecc greater than mean $\Delta$ Ecc + one standard deviation (SD) and "definite" improvement as Ecc improvement greater than mean $\triangle \mathrm{Ecc}-\mathrm{SD}$. Comparisons of continuous variables between the "definite" myocardial functional deterioration group and the remainder group of participants were performed using t-tests and those of discrete variables by chi-square tests. Prevalence of "definite" Ecc deterioration group was compared between groups with CRP $>3 \mathrm{mg} / \mathrm{L}$ and $\leq 3 \mathrm{mg} / \mathrm{L}$ using chi-square test. Comparison of nonparametric parameters was performed using MannWhitney $\mathrm{U}$ test. Both multivariable linear regression analysis of $\Delta \mathrm{Ecc}$ and logistic regression analysis for "definite" Ecc deterioration were performed to evaluate the predictability of baseline parameters. We evaluated and compared the fitness, statistical results and interpretation of findings obtained using the following models. Model 1 included age, gender and ethnicity, Model 2 included systolic blood pressure, heart rate, diabetes, smoking status, body mass index, current medications (antihypertensive medication, aspirin and statin) and glomerular filtration rate in addition to Model 1 variables, while Model 3 included LV mass, coronary calcium score, interim myocardial infarction and coronary revascularization in addition to Model 2 . Because $\Delta$ Ecc was significantly correlated with baseline Ecc, we adjusted for baseline Ecc in Model 4. To examine whether the results were independent of interim coronary events, we repeated the analyses after exclusion of participants who experienced interim coronary events. All statistical analyses were performed using SPSS (version 15.0, SPSS, Inc., Chicago, IL, USA) and we considered two sided P-values $<0.05$ to be significant.

\section{Results}

\section{Baseline CRP and participant characteristics}

Among the 1,773 subjects who were enrolled in MESA baseline ancillary tagging study, 851 participants underwent repeated tagged MRI. Among them 62 cases were excluded because 
of noise, mis-registration of tag, different tagging sequence and unreadable images by analyzing software. Four cases were lost due to no CRP data. Finally total 785 cases had both CRP value and good quality mid-LV slice strain curve from both baseline and followup. Mean age was $64 \pm 9$ years and 386 participants were women (49\%). The median CRP concentration was $1.7 \mathrm{mg} / \mathrm{L}$ (interquartile range 0.8 to $3.8 \mathrm{mg} / \mathrm{L}$ ) and women had higher CRP than men $(2.42 \mathrm{mg} / \mathrm{L}$ vs. $1.25 \mathrm{mg} / \mathrm{L}, \mathrm{p}<0.001)$. The mean interval between the baseline and follow-up MRI examinations was 1,713 \pm 95 days. During the follow-up period, six patients suffered myocardial infarction (time to interim myocardial infarction was $1,390 \pm$ 444 days) and 18 underwent coronary revascularization (time to revascularization 1,176 \pm 506 days) with 3,690 person-years of follow-up. Table 1 shows the baseline characteristics and LV indices for each sex-specific CRP quartile. The higher CRP quartile subgroup was associated with higher body mass index, higher prevalence of hypertension, smoker, African American, Hispanic, and higher LV mass, Chinese Americans and statin users were associated with lower CRP quartiles.

\section{Longitudinal myocardial functional changes}

Temporal change in circumferential myocardial shortening was used to index changes in myocardial function across time. Negative $\Delta$ Ecc values reflect functional improvement while positive $\Delta$ Ecc indicates functional deterioration. Mean mid-ventricular Ecc did not significantly change for the entire study group during the follow-up period (from $-17.8 \pm 2.6 \%$ to $-17.9 \pm 2.8 \%, \mathrm{P}=0.26$ ), and average $\Delta \mathrm{Ecc}$ (i.e. Ecc at follow-up - Ecc at baseline) was $-0.1 \pm 3.3 \%$. Therefore we considered $\Delta \mathrm{Ecc}>3.2 \%$ (Ecc deterioration more than one SD) as "definite" myocardial functional deterioration and $\Delta \mathrm{Ecc}<-3.4 \%$ as "definite" Ecc improvement. The "definite" deterioration group (N=112) had higher baseline CRP. The prevalence of current smokers was significantly higher and the prevalence of Chinese was significantly lower in the "definite" deterioration group (Table 2). Higher body mass index was related to "definite" deterioration group in men (OR1.1, 1.03-1.18, $\mathrm{p}=0.004)$ but not in women $(1.04,0.99-1.11, \mathrm{p}=0.13)$. Hypertension $(\mathrm{OR} 1.64,1.09-2.48$, $\mathrm{p}=0.018)$ and angiotensin converting enzyme inhibitor users (OR 2.02, 1.21-3.38, $\mathrm{p}=0.008)$ were significantly related to "definite" Ecc improvement.

\section{Predictors of regional myocardial functional deterioration}

Baseline CRP was weakly but significantly correlated with change in myocardial shortening ( $\triangle \mathrm{Ecc}, \mathrm{r}=0.11, \mathrm{p}=0.002$ ) indicating that increased CRP is associated with myocardial functional reduction. In univariate analysis, higher CRP was significantly correlated with "definite" myocardial functional deterioration (OR 1.07 per $1 \mathrm{mg} / \mathrm{L}$ increase, 95\% CI 1.03$1.11, \mathrm{p}<0.001)$. Because $\Delta \mathrm{Ecc}$ was significantly correlated with baseline Ecc $(\mathrm{r}=-0.59$, $\mathrm{P}<0.001$ ), we re-analyzed the data after adjusting for baseline Ecc. Higher CRP, indexed LV mass, male gender, current smoking and diastolic blood pressure were all significantly correlated with the myocardial functional deterioration after adjustment for baseline Ecc values both in categorical and continuous variable analyses (Table 3). Participants with $\mathrm{CRP}>3 \mathrm{mg} / \mathrm{L}$ had higher prevalence of Ecc deterioration compared to participants with CRP $\leq 3 \mathrm{mg} / \mathrm{L}(20 \%$ vs. $12 \%, \mathrm{p}=0.003)$.

\section{CRP as an independent predictor of regional myocardial functional deterioration}

In linear regression analysis, higher CRP was related to functional deterioration independent of age, gender and ethnicity $(B=0.08 ; \Delta$ Ecc change per $1 \mathrm{mg} / \mathrm{L}$ CRP change, $95 \%$ CI 0.04 $0.13, \mathrm{p}<0.001$, Model 1). In Model 2, which included systolic blood pressure, heart rate, diabetes, smoking status (never, former, current), body mass index, current medication use (antihypertensive therapy, aspirin, and statins) and glomerular filtration rate in addition to Model 1 covariates, higher CRP was also related to Ecc deterioration $(B=0.10,0.05-0.14$, $\mathrm{p}<0.001$ ). In Model 3, we included indices of subclinical cardiovascular disease (LV mass, 
presence of coronary calcium) as well as interim myocardial infarction and coronary revascularization in addition to Model 2 covariates. Higher CRP remained related to Ecc deterioration $(\mathrm{B}=0.09,0.05-0.14, \mathrm{p}<0.001)$. Moreover, the relationship remained statistically significant after adjustment for baseline Ecc in Model $4(\mathrm{~B}=0.06,0.02-0.10$, $\mathrm{p}=0.002$ ). Even after systolic blood pressure was replaced by diastolic blood pressure, relation between CRP and Ecc change remained significant. Finally, in a sensitivity analysis, after exclusion of participants with interim myocardial infarction or coronary revascularization, higher CRP was still significantly related to Ecc deterioration. Importantly also, in logistic regression analysis, higher CRP was significantly correlated with "definite" myocardial deterioration using the same models (Table 4).

\section{Discussion}

In this study, we found that elevated levels of CRP were significantly associated with future regional myocardial functional deterioration in asymptomatic individuals independent of coronary artery disease and its clinical consequences. To the best of our knowledge, the present study is the first longitudinal population study to demonstrate the relationship between inflammation and progressive myocardial dysfunction. Our results might provide mechanistic support to the well established association of elevated CRP with incident HF, suggesting therefore that inflammation may have an active role in the pathogenesis of HF.

\section{Age-related changes in circumferential shortening}

Myocardial circumferential strain is a sensitive indicator of ventricular function and is more reliable than indices of global ventricular function for the assessment of myocardial mechanics. ${ }^{7}$ Detection of subclinical myocardial dysfunction could potentially identify asymptomatic individuals at high risk for the development of HF which could be systolic or diastolic. ${ }^{13}$ We found that a subset of MESA participants with heightened inflammation as surrogated by increased baseline CRP had experienced subsequence deterioration of circumferential mid-myocardial function during the five years of this study, despite the absence of interim myocardial infarction or other clinical manifestations of coronary artery disease. Such deterioration could have been caused by microvascular dysfunction, age related subclinical interstitial fibrosis, or worsening of calcium-ATP metabolism accompanied by adverse ventricular remodeling, among other potential mechanisms. ${ }^{14}$ Meanwhile, many participants exhibited an increase in circumferential shortening during the five years of follow-up. This might be explained by different loading conditions between the baseline and follow-up examinations, measurement error or true functional improvement due to therapy, life style changes or compensatory hypertrophy to maintain ejection fraction in the face of increased blood pressure and vascular stiffness. ${ }^{15,16}$ In our results, hypertensive people, especially angiotensin converting enzyme inhibitor users were significantly related to Ecc improvement, which supports presence of compensatory mechanism in preclinical hypertensive population. ${ }^{15}$ In order to reduce the effects of different loading conditions as well as other sources of variability at each examination, we considered "definite" regional myocardial functional deterioration as Ecc deterioration greater than one SD in average myocardial circumferential strain at the mid-LV crosssectional level. In addition, we also analyzed change in myocardial strain as a continuous variable using linear regression analysis.

\section{Inflammation and regional myocardial dysfunction}

In our study, the group with the highest CRP quartile exhibited significantly higher prevalence of current smoking, hypertension, higher body mass index and LV mass. This finding might suggest that CRP would simply be a mediator of other risk factors thought to be associated with myocardial functional deterioration. However, in multivariable analyses, 
elevated CRP was correlated with future Ecc deterioration after adjustment for these CRPinfluencing conditions. Therefore, it is possible that, regardless of the origin of elevated CRP, long-standing exposure to chronic inflammation might actively contribute to progressive myocardial dysfunction through activation of the complement system, promotion of endothelial dysfunction, or direct myocyte injury. ${ }^{6,}{ }^{17}$ In univariate analyses, male gender, current smoking and higher LV mass index, all of which are related to the development of silent myocardial infarction, exhibited significant direct relationships with myocardial functional deterioration. On the other hand, higher CRP remained significantly associated with myocardial functional deterioration after adjustment for coronary risk factors and coronary calcification as well as interim symptomatic myocardial infarction and coronary revascularization. While it is still possible that asymptomatic microinfarcts contribute to progressive myocardial dysfunction among asymptomatic individuals, as we did not assess the presence of myocardial scar with contrast MRI in this study. Nevertheless, if significant in magnitude, such contribution appears to be at least in part mediated by inflammation.

There are controversies regarding beneficial effects of anti-inflammatory agent on HF. TNFa inhibitors failed to achieve beneficial effects on HF. ${ }^{18}$ However, statin therapy achieved some beneficial effects on higher CRP subgroup by actively reducing CRP level in advanced HF patients in CORONA study. ${ }^{19}$ This finding raised the question about in which stage inflammation can contribute to HF or whether there is any difference among antiinflammatory agents. Our study could neither approach which inflammatory stage can affect myocardial functional change nor effects of anti-inflammatory agents. However, based on our results, CRP, downstream inflammatory biomarker, is at least related to progressive myocardial function in subclinical stage, suggesting elevated CRP is not just redundant of HF. Our results support the concept that, even among asymptomatic subjects, risk stratification based on CRP might be useful for assessing the risk and progression of cardiovascular disease in general and of myocardial dysfunction ${ }^{5}$. Our study results provide a link between observed cross-sectional relationships and the development of HF.

\section{Methodological consideration and study limitations}

This study has some limitations. First, we analyzed high sensitive CRP values assessed only at baseline examination. CRP values can vary according to conditions such as systemic or local inflammation; however, CRP has been shown to be relatively stable in the absence of active inflammation, and less than $5 \%$ of the participants in the present study had levels higher than $10 \mathrm{mg} / \mathrm{L}$, suggesting active systemic inflammation. ${ }^{20}$ Second, the myocardial region of interest in which we measured myocardial strain may not exactly match between the two MRI tagging examinations from a single individual. In this regard, specific efforts were taken during the follow-up examination by using the baseline study for guiding the same anatomic location. Third, participants in this study have lower body mass index and lower CRP compared to whole MESA population possibly from selection bias for MRI and higher prevalence of Chinese in this study compared to whole MESA population (18\% vs. $12 \%)$. However these limitations might not affect much on the results as we used both categorical and continuous end-point, number of Chinese population was still lower than other ethnicity and possible exclusion of highly obese participants who were not to fit in scanner was unavoidable and infrequent.

\section{Conclusion}

We found that elevated high sensitive CRP is an independent predictor of myocardial functional deterioration among asymptomatic adult individuals without history of prior heart disease. This finding may in part explain the previously documented association of CRP with incident HF in population based studies including the MESA cohort. They also support 
continued research on the impact of anti-inflammatory therapies ${ }^{19}$, for prophylaxis of myocardial functional deterioration.

\section{Acknowledgments}

This study was supported by the National Heart, Lung, and Blood Institute grant (RO1-HL66075-01) and the MESA study contracts (NO1-HC-95162, NO1-HC-95168, and NO1-HC-95169).

\section{References}

1. Anand IS, Latini R, Florea VG, et al. C-reactive protein in heart failure: prognostic value and the effect of valsartan. Circulation. 2005; 112:1428-34. [PubMed: 16129801]

2. Vasan RS, Sullivan LM, Roubenoff R, et al. Framingham Heart Study. Inflammatory markers and risk of heart failure in elderly subjects without prior myocardial infarction: the Framingham Heart Study. Circulation. 2003; 107:1486-91. [PubMed: 12654604]

3. Bahrami H, Bluemke DA, Kronmal R, et al. Novel metabolic risk factors for incident heart failure and their relationship with obesity: The MESA (Multi-Ethnic Study of Atherosclerosis) Study. J Am Coll Cardiol. 2008; 51:1775-83. [PubMed: 18452784]

4. Willerson JT, Ridker PM. Inflammation as a cardiovascular risk factor. Circulation. 2004; 109:II2II10. [PubMed: 15173056]

5. Rosen BD, Cushman M, Nasir K, et al. Relationship between C-reactive protein levels and regional left ventricular function in asymptomatic individuals: the Multi-Ethnic Study of Atherosclerosis. J Am Coll Cardiol. 2007; 49:594-600. [PubMed: 17276184]

6. Prabhu SD. Cytokine-induced modulation of cardiac function. Circ Res. 2004; 95:1140-53. [PubMed: 15591236]

7. Yeon SB, Reichek N, Tallant BA, et al. Validation of in vivo myocardial strain measurement by magnetic resonance tagging with sonomicrometry. J Am Coll Cardiol. 2001; 38:555-61. [PubMed: 11499752]

8. Bild DE, Bluemke DA, Burke GL, et al. Multi-ethnic study of atherosclerosis: objectives and design. Am J Epidemiol. 2002; 156:871-81. [PubMed: 12397006]

9. Castillo E, Osman NF, Rosen BD, et al. Quantitative assessment of regional myocardial function with MR-tagging in a multi-center study: interobserver and intraobserver agreement of fast strain analysis with Harmonic Phase (HARP) MRI. J Cardiovasc Magn Reson. 2005; 7:783-91. [PubMed: 16358393]

10. Natori S, Lai S, Finn JP, et al. Cardiovascular function in multi-ethnic study of atherosclerosis: normal values by age, sex, and ethnicity. AJR Am J Roentgenol. 2006; 186:S357-65. [PubMed: 16714609]

11. Garot J, Bluemke DA, Osman NF, et al. Fast determination of regional myocardial strain fields from tagged cardiac images using harmonic phase MRI. Circulation. 2000; 101:981-8. [PubMed: 10704164]

12. Fernandes VR, Polak JF, Cheng S, et al. Arterial stiffness is associated with regional ventricular systolic and diastolic dysfunction: the Multi-Ethnic Study of Atherosclerosis. Arterioscler Thromb Vasc Biol. 2008; 28:194-201. [PubMed: 17962621]

13. McMurray JV, McDonagh TA, Davie AP, Cleland JG, Francis CM, Morrison C. Should we screen for asymptomatic left ventricular dysfunction to prevent heart failure? Eur Heart J. 1998; 19:8426. [PubMed: 9651707]

14. Lakatta EG. Arterial and cardiac aging: major shareholders in cardiovascular disease enterprises, III: cellular and molecular clues to heart and arterial aging. Circulation. 2003; 107:490-7. [PubMed: 12551876]

15. Wang J, Khoury DS, Yue Y, Torre-Amione G, Nagueh SF. Preserved left ventricular twist and circumferential deformation, but depressed longitudinal and radial deformation in patients with diastolic heart failure. Eur Heart J. 2008; 29:1283-9. [PubMed: 18385117] 
16. Cheng S, Fernandes VR, Bluemke DA, McClelland RL, Kronmal RA, Lima JA. Age-related left ventricular remodeling and associated risk for cardiovascular outcomes The Multi-Ethnic Study of Atherosclerosis. Circ Cardiovasc Imaging. 2009; 2:191-8. [PubMed: 19808592]

17. Verma S, Li SH, Badiwala MV, et al. Endothelin antagonism and interleukin-6 inhibition attenuate the proatherogenic effects of C-reactive protein. Circulation. 2002; 105:1890-6. [PubMed: 11997273]

18. Chung ES, Packer M, Lo KH, Fasanmade AA, Willerson JT. Randomized, Double-Blind, PlaceboControlled, Pilot Trial of Infliximab, a Chimeric Monoclonal Antibody to Tumor Necrosis Factor$\mathrm{a}$, in Patients With Moderate-to-Severe Heart Failure: Results of the Anti-TNF Therapy Against Congestive Heart failure (ATTACH) Trial. Circulation. 2003; 107:3133-40. [PubMed: 12796126]

19. McMurray JJ, Kjekshus J, Gullestad L, et al. CORONA Study Group. Effects of statin therapy according to plasma high-sensitivity C-reactive protein concentration in the Controlled Rosuvastatin Multinational Trial in Heart Failure (CORONA): a retrospective analysis. Circulation. 2009; 120:2188-96. [PubMed: 19917888]

20. Du Clos TW. Function of C-reactive protein. Ann Med. 2000; 32:274-8. [PubMed: 10852144] 


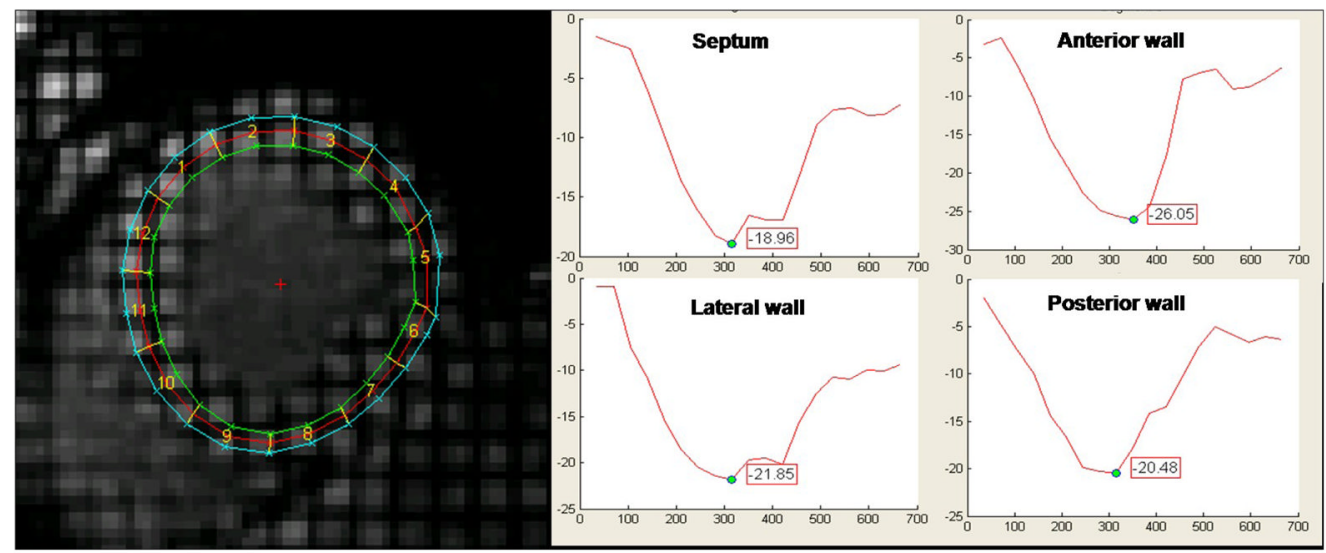

Figure 1.

Tagged MRI Study with a sample circumferential strain curve (\%strain on y-axis; in milliseconds on $\mathrm{x}$-axis) in a cross section of $\mathrm{LV}$ 
Table 2

Comparisons of baseline demographics, risk factors, and medications between the "definite" deterioration group and the other group.

\begin{tabular}{lccc}
\hline & Definite Deterioration $(\mathbf{n = 1 1 2})$ & Remainder $(\mathbf{n}=\mathbf{6 7 3})$ & $\mathbf{P}$ \\
\hline Age, yr & $64.5 \pm 9.9$ & $63.7 \pm 9.2$ & 0.45 \\
Female, $\mathrm{n}(\%)$ & $50(45)$ & $336(50)$ & 0.30 \\
Body mass index, $\mathrm{kg} / \mathrm{m}^{2}$ & $27.4 \pm 5.1$ & $27.8 \pm 4.6$ & 0.48 \\
Heart rate, bpm & $62.4 \pm 9.9$ & $62.3 \pm 9.2$ & 0.96 \\
Hypertension, $\mathrm{n}(\%)$ & $54(48)$ & $313(47)$ & 0.74 \\
Diabetes, $\mathrm{n}(\%)$ & $14(12)$ & $81(12)$ & 0.89 \\
Ethnicity, $\mathrm{n}(\%)$ & & & \\
$\quad$ Non-Hispanic white & $41(37)$ & $200(30)$ & 0.14 \\
$\quad$ Chinese-American & $12(11)$ & $127(19)$ & 0.04 \\
$\quad$ African-American & $36(32)$ & $164(24)$ & 0.08 \\
$\quad$ Hispanic & $23(21)$ & $182(27)$ & 0.15 \\
Current smoking, $\mathrm{n}(\%)$ & $19(17)$ & $60(9)$ & 0.008 \\
LDL-cholesterol, mg/dL & $118.7 \pm 32.0$ & $118.9 \pm 31.5$ & 0.95 \\
HDL-cholesterol, mg/dL & $50.6 \pm 14.5$ & $51.1 \pm 15.0$ & 0.76 \\
Urine albumin/creatinine, mg/g & $12.5 \pm 20.4$ & $16.3 \pm 46.2$ & 0.40 \\
CRP ${ }^{*}$, mg/L & $2.4(1.0-5.4)$ & $1.6(0.8-3.5)$ & 0.005 \\
Presence of coronary calcium,n(\%) & $65(58)$ & $346(51)$ & 0.19 \\
LV ejection fraction,\% & $70.3 \pm 7.0$ & $69.1 \pm 7.5$ & 0.12 \\
LV mass index, g/m ${ }^{2}$ & $80.0 \pm 16.6$ & $77.6 \pm 16.4$ & 0.17 \\
\hline * & & & \\
Median (interquartile range); & & & \\
\hline
\end{tabular}

$\mathrm{CCB}$, calcium channel blocker; ACE, angiotensin-converting enzyme; ARB, angiotensin receptor blocker;LDL, low-density lipoprotein; HDL, high density lipoprotein; CRP, C-reactive protein; LV, left ventricular 
